\title{
Spinal pain: current understanding, trends, and the future of care
}

This article was published in the following Dove Press journal:

Journal of Pain Research

23 October 2015

Number of times this article has been viewed

\section{Gregory F Parkin-Smith' Lyndon G Amorin-Woods ${ }^{2-4}$ \\ Stephanie J Davies ${ }^{5-7}$ \\ Barrett E Losco ${ }^{8}$ \\ Jon Adams ${ }^{9,10}$}

'General Practice, Surgery 82, Busselton, WA, Australia; ${ }^{2}$ School of Health Professions, Murdoch University, Murdoch, WA, Australia; ${ }^{3}$ Chiropractors' Association of Australia, Nedlands, WA, Australia; ${ }^{4}$ ACORN Project, ${ }^{5}$ WA Specialist Pain Services, WA, Australia; ${ }^{6}$ School of Physiotherapy, Curtin University, Bentley, WA, Australia; ' 5 chool of Medicine and Pharmacology, University of Western Australia, Crawley, WA, Australia; ${ }^{8}$ Murdoch University, Murdoch, WA, Australia; ${ }^{9}$ Faculty of Health, University of Technology Sydney, Ultimo, NSW, Australia; ${ }^{10}$ Australian Research Centre in Complementary and Integrative Medicine, University of Technology Sydney, Ultimo, NSW, Australia
Correspondence: Gregory F Parkin-Smith General Practice, Surgery 82, 82 Bussell Highway, Busselton, WA 6280, Australia Email g.parkin.smith@gmail.com

\begin{abstract}
This commissioned review paper offers a summary of our current understanding of nonmalignant spinal pain, particularly persistent pain. Spinal pain can be a complex problem, requiring management that addresses both the physical and psychosocial components of the pain experience. We propose a model of care that includes the necessary components of care services that would address the multidimensional nature of spinal pain. Emerging care services that tailor care to the individual person with pain seems to achieve better outcomes and greater consumer satisfaction with care, while most likely containing costs. However, we recommend that any model of care and care framework should be developed on the basis of a multidisciplinary approach to care, with the scaffold being the principles of evidence-based practice. Importantly, we propose that any care services recommended in new models or frameworks be matched with available resources and services - this matching we promote as the fourth principle of evidence-based practice. Ongoing research will be necessary to offer insight into clinical outcomes of complex interventions, while practice-based research would uncover consumer needs and workforce capacity. This kind of research data is essential to inform health care policy and practice.
\end{abstract}

Keywords: back pain, pain management, combined modality therapy, patient care team

\section{Background}

A large body of research now exists related to the treatment of nonmalignant spinal pain, with literally hundreds of reports on clinical trials showing the benefit of the treatments under investigation. For example, Hurwitz ${ }^{1}$ indicated that there were over 200 reports on clinical trials related to spinal manipulative therapy and exercise for low back pain alone, with several other conservative treatments also being recommended in published clinical guidelines ${ }^{2-4}$ and systematic reviews for nonmalignant spinal pain. ${ }^{5-7}$ Well-known and widely accepted treatments, with at least a moderate benefit, ${ }^{8}$ include advice and education, ${ }^{9}$ returning to work, ${ }^{10}$ being active,${ }^{11,12}$ exercise,${ }^{13,14}$ and manual and manipulative therapy, among others. ${ }^{15,16}$ Nonmalignant neck and thoracic pain should also be mentioned here as very common problems, again with the research evidence offering insight that similar conservative treatment(s) have a beneficial effect. ${ }^{17-19}$ In fact, a cross-sectional, nation-wide survey of the Danish population showed remarkably similar patterns in pain reporting and the consequences of pain for the three spinal regions (cervical, thoracic, and lumbosacral), respectively. ${ }^{20}$ The implication is that nonmalignant spinal pain may be considered as essentially the same condition regardless of the region of the spine involved. Therefore, we can reasonably speculate that 
interventions shown to be of benefit for low back pain are also likely to be effective for pain in other areas of the spine.

It is now questionable whether further research into existing individual treatments for nonmalignant spinal pain would add to our current understanding. Updates of wellknown, published clinical guidelines and systematic reviews encourage specific effective treatments that were shown to be useful for the specific cohort of patients recruited to each of the reviewed studies. Furthermore, we know from experience and published trials ${ }^{21-23}$ that health care practitioners, quite reasonably, combine treatments in an attempt to offer patients the best possible outcomes with respect to their pain. Often, treatments are prescribed or delivered together, such as simple analgesics and advice, or a combination of education, exercise, and manual and manipulative therapy. The aim is to take advantage of the plausible synergistic effects of these treatments, often with greater patient satisfaction with care. ${ }^{24,25}$ For example, in the United Kingdom Back Pain Exercise and Manipulation (BEAM) trial exploring low back pain of more than 4 weeks duration, the number needed to treat for spinal manipulation for nonmalignant low back pain is 5.4 and for exercise is 5.0, thereby presenting either treatment as an attractive option for clinicians, patients, and policy makers. Yet, if a package of care is used, such as combining spinal manipulation followed by exercise, this results in a number needed to treat of $3.3,{ }^{26}$ which is even better.

Spinal pain, particularly persistent pain, is a complex phenomenon, and it is very real to the person experiencing it. Persistent pain has both physical and psychosocial components. ${ }^{27,28}$ Accordingly, there is an evolving trend toward matching care to both the physical and psychosocial components of a patient's pain experience. This implies determining the patient's care needs, be these physical and/or psychosocial, and matching them with the best available evidence-based active and passive treatments. In conjunction, care services and access to these services need to be identified and developed to meet the patient's care needs. The end product is a care package or protocol that is tailored to the individual patient, ranging from low to high levels of care complexity, with a view to gaining the best possible outcomes. This approach to care would be achieved through multidisciplinary health care teams and utilizing a multimodal, biopsychosocial approach to care - this approach now being reflected in emerging innovative Models of Care (MoC) and Care Frameworks.

The health care demand issues facing developed countries are well-known, particularly related to the needs of an aging population, chronic disease, sometimes ailing workforce, and dramatic cost inflation. ${ }^{29}$ Therefore, the emerging focus in health care is to have care services delivered using mechanisms or strategies that are cost efficient and evidence based, ${ }^{25,30}$ wherever possible, while engaging with the broadest applicable workforce. In the past, clinical guidelines have attempted to do this, but, as stated in the Australian National Health and Medical Research Council (NHMRC) report ${ }^{31}$ on the utilization and adherence to clinical guidelines:

Governments have funded $22 \%$ of the guidelines in this report, yet there remains a demonstrable lack of coordination in the way guidelines are prioritised and commissioned in key clinical areas ... [and] ... Effective implementation of guidelines still remains a key challenge for guideline developers and funders ....

The message is that nonadherence to guidelines have led to limited implementation, which is speculated to have significant cost and resource implications for health care systems.

The problems with adherence and implementation of clinical guidelines are well known. ${ }^{32}$ This does not imply that guidelines are of poor quality or that their outcomes are meaningless. On the contrary, the problem lies with the implementation of guideline recommendations in real-life practice, not the quality of the guideline in itself. Indeed, another neglected aspect is acceptability of guideline recommendations to the end user, be it the clinician, manager, policy maker, academic, or consumer, respectively. ${ }^{33,34} \mathrm{~A}$ fresh model is needed that goes beyond clinical guidelines to facilitate the adherence and implementation of recommended care, where the content, layout, format, and illustrations are tailored to the user and their context.

In addition, reviews and guidelines offer little insight into the multidisciplinary activities necessary, within the context of the biopsychosocial model of pain management, to successfully deal with the problem of persistent spinal pain. With the spotlight now on multidisciplinary care, we highlight the necessary involvement of various health care providers. This is auspicious, since this broadens the workforce capacity to manage the growing problem of spinal pain through teamwork and task substitution, thereby potentially avoiding the huge cost and effort to train new graduates and future practitioners in the area of spinal pain.

The purpose of this paper, drawing specifically upon the Australian context and experience, is to offer the reader a review of the care services and pain management approach that is likely to facilitate the successful management of nonmalignant spinal pain. To achieve this aim, the objectives are to offer: 1) a précis of our contemporary understanding of 
the treatments for nonmalignant low back pain, supported by the best available research evidence; 2) a description of the biopsychosocial model, which is the emerging scaffold for current care services; 3 ) to show how the biopsychosocial approach may be represented in a Model of Care; 4) to briefly describe how the whole workforce relevant to spinal pain may be rallied to provide care services; and 5) to share our insights into the potential features of future care services and associated research.

We acknowledge that pain states may be acute, subacute, and chronic. However, it is beyond the scope of this paper to provide an account of each of these categories, even though these categories share many similar characteristics, ${ }^{35}$ because the ideas as set out in this paper may be generalized across all three categories.

\section{Update on the research evidence}

Recommendations on treatments for acute and chronic spinal pain are published elsewhere in numerous reviews and clinical guidelines. We emphasize that guidelines seldom offer a full account of either 1) exactly how each recommended treatment should be applied, or 2) if a specific combination of these treatments would be more useful than another. In this regard, more recent research is showing that treatments previously believed to be equivocal are now emerging as clearly beneficial. For example, in a randomized controlled trial testing guidelines-based care, Bishop et $\mathrm{al}^{36}$ showed that significantly greater improvement for acute mechanical low back pain of 16 weeks, or less, was achieved with spinal manipulative therapy compared with usual medical care. Patients receiving usual medical care had inferior functional outcomes in conjunction with higher rates of prescribed opioid analgesics (80\%). The COST B13 European guidelines ${ }^{37}$ differentiate between supporting the use of weak opioids (eg, tramadol), for acute and chronic back pain, and do not mention strong opioids for acute back pain. The COST B13 guideline indeed comments on the limited evidence for strong opioids in chronic back pain.

There is strong evidence that weak opioids (eg, tramadol) relieve pain and disability in the short-term in chronic low back pain patients (level A). There is limited evidence that strong opioids relieve pain in the short-term in chronic low back pain patients (level C).

Furthermore, medically managed patients received a high percentage $(60 \%)$ of guideline-discordant treatment, like bed rest, X-rays, and back supports. Goertz et $\mathrm{al}^{38}$ demonstrated that spinal manipulative therapy in conjunction with standard medical care offers a significant advantage for decreasing pain and improving physical functioning when compared with standard care alone for men and women between 18 and 35 years of age with acute low back pain. In contrast, Hay et $\mathrm{al}^{39}$ showed that a brief pain management program for back pain delivered by appropriately trained clinicians offers an alternative to physiotherapy-incorporating manual therapy and could provide an efficient first-line approach for subacute low back pain in primary care. The inferences from these trials ${ }^{37-39}$ are that there are various effective treatments for nonmalignant spinal pain, and that a multimodal approach, combining interventions, is likely to yield better outcomes - in this case patient education with both active and passive treatments. Such studies, among others, also firmly support the early access of patients with nonmalignant spinal pain to assessment, with an emphasis on triage and diagnosis, and appropriate treatment, to achieve the best possible outcome. ${ }^{37-39}$

Fortuitously, research exploring the outcomes of a combination of treatments is now emerging in the health care literature and in practice across many health care sectors. For example, a before-and-after clinical trial of a multimodal treatment program for hip and knee arthroplasty led to a shorter duration of stay when compared to usual care. ${ }^{40}$ Of course, we acknowledge that care pathways and treatment protocols have been around for some years in specific health care disciplines, including pain medicine, ${ }^{41}$ but only more recently have there been earnest attempts to examine these protocols in definitive studies using appropriate research design to test these complex interventions. Patrick et $\mathrm{al}^{42}$ showed that multidisciplinary treatments for chronic pain are superior to no treatment, waiting list (patients waiting to be consulted by a clinician, not yet having received any treatment), as well as single-discipline treatments such as medical treatment or physical therapy. Moreover, the effects appeared to be stable over time. The beneficial effects of multidisciplinary treatment were not limited to improvements in pain, mood, and cognitive interference (unwanted and often disturbing thoughts that play an important role in stress, poor performance, slow learning, social maladjustment), but also extended to behavioral variables such as return to work or use of the health care system.

We propose that multimodal, multidisciplinary care has a beneficial effect on nonmalignant spinal pain and is an approach to care worth pursuing. For example, Monticone et $\mathrm{al},{ }^{43}$ in a clinical trial of a multidisciplinary rehabilitation program on disability, kinesiophobia, catastrophizing, pain, quality of life, and gait disturbances in patients with chronic low back pain, showed that a multidisciplinary rehabilitation program including cognitive behavioral therapy was superior 
to an exercise program. Moradi et $\mathrm{al}^{44}$ showed that multidisciplinary treatment ameliorates pain, improves both functional restoration and quality of life, with medium to high effect sizes, even for patients with a long history of chronic back pain. Effect sizes are higher than for monodisciplinary treatments, and treatment effects remained stable at 6-month follow-up. Moradi et $\mathrm{al}^{44}$ conclude that multidisciplinary treatment is vital for the management of patients with chronic low back pain.

Published research protocols of trials currently being implemented promise exciting outcomes. In a proposed Danish study, guidelines on low back pain management are being tested in a clinical trial. ${ }^{45}$ The expectation is that the implementation strategy will reduce the number of patients referred to secondary care, and that the additional upfront cost of extended implementation will be counterbalanced by improvements in clinical practice and patient-related outcomes, thereby rendering the strategy cost efficient. In another proposed randomized controlled clinical trial in working-age patients with chronic low back pain, ${ }^{46}$ three treatment strategies are to be compared: 1) intensive and multidisciplinary program conducted in a rehabilitation center, 2) less intensive outpatient program conducted by a private physiotherapist, and 3) a mixed strategy combining the same outpatient program with a multidisciplinary intervention - the hypothesis is that a multidisciplinary approach will be the key feature of success in reducing social and occupational impairment. Therefore, it may be possible to achieve the same positive results with less intensive strategies if a multidisciplinary approach is maintained.

The clear message from current and emerging research is that multidisciplinary care is very likely to have a major positive impact on clinical outcomes, patient satisfaction with care, and cost savings. In this regard, development of $\mathrm{MoC}$ or care service frameworks needs to incorporate the principles of evidence-based health care, which are: 1) use of the best available research evidence, 2) inclusion of clinical expertise, and 3) acknowledgment of consumer preferences. ${ }^{47}$ We also propose the fourth "leg" or principle of evidence-based health care, which should be the careful consideration of available resources - (sustainable) funding, resources, access, and workforce capacity - otherwise even a well-designed framework or model would neither be feasible to implement nor acceptable to end users.

\section{The multidimensional nature of pain}

The International Association for the Study of Pain defines pain as "an unpleasant sensory and emotional experience associated with actual or potential tissue damage, or described in terms of such damage" ${ }^{48}$ Therefore, the concept of pain can be complex and difficult to grasp. As a multifaceted phenomenon, pain requires a versatile approach to care, with a view to achieve the best possible outcomes. ${ }^{49}$ Nonmalignant spinal pain, particularly persistent pain, is no different and should be considered under the umbrella of the pain conundrum. Unraveling the puzzle of pain starts with each person who has persistent pain becoming aware of the interlinking contribution of injury pain (thought of as nociception), neuropathic pain, inflammatory pain, and increasingly, the likelihood of immunoreactive component(s). ${ }^{50}$ The emphasis here is that pain is complex and requires a likewise multifaceted approach to care. We feel that a patient-specific approach drawing upon the expertise of multiple health care disciplines is emerging as the best practice approach.

The biopsychosocial model of care, which is characterized by multidisciplinary, multimodal care, is now a widely accepted strategy for the management of persistent pain. ${ }^{27,28}$ For example, a systematic review suggested that there was moderate evidence that multidisciplinary rehabilitation was effective for subacute low back pain. ${ }^{51}$ This multidisciplinary approach with "whole person engagement" is now gaining acceptance as an important way of connecting with and managing persons with pain because there are interdependent relationships between the physical and psychological factors associated with pain ${ }^{52}$ that require a holistic approach. Epidemiological studies have also highlighted that the psychosocial factors linked with low back pain can be used as prognostic indicators, eg, depression. ${ }^{53,54}$ These studies highlight the need to target care toward both the deleterious physical and psychosocial aspects of pain.

The management of pain is a bit like a jigsaw puzzle, with medications or procedures only representing one of the pieces of a multipiece jigsaw of cocare, multimodal options. So far, current care services may not be measuring all the pieces of this jigsaw puzzle entirely. For example, Froud et $a 1,{ }^{55}$ in a systematic review and meta-analysis, indicated that the impact of the experience of persistent pain on the affected person is profound, yet they found that despite the suffering being significant, core outcomes often did not capture what was important. Froud et $\mathrm{al}^{55}$ suggest a move toward a biopsychosocial model that covers core sets of relevant outcomes. Cocare, via multidisciplinary working teams, ${ }^{56}$ implies coordinated care between knowledgeable consumers and a range of health care providers, each representing pieces of the puzzle, ${ }^{57}$ with a view to bring the pieces together and develop a beneficial management plan tailored to the individual person with pain. 
Now, more than ever, coordinated action is needed to plan for the short- and long-term care needs of persons with pain. In the past, care services for pain have been quite discrete and have not taken full advantage of the benefits of the multidisciplinary approach to care. Contemporary management of pain is evolving toward this multidisciplinary way, with a view to obtain better outcomes. However, there are still barriers, including local health care politics, workforce capacity, funding mechanisms, and the gap in explicit $\mathrm{MoC}$ that are designed to guide care in real-world practice.

\section{The economic cost of spinal pain}

The importance of addressing spinal pain in a cost-effective and clinically appropriate manner is illustrated in a series of studies emerging from the Global Burden of Disease 2010 Project. ${ }^{58}$ It is well-known that musculoskeletal conditions, such as low back pain, neck pain, and arthritis, affect more than 1.7 billion people worldwide and are set to become more prevalent in the developed world with a growing aging population. ${ }^{59}$ Pain is now recognized worldwide as an area of health care need and, in Australia, it is emerging as a national priority ${ }^{60}$ Chronic pain is the third most costly condition in annual health expenditure in Australia ( $\$ 34$ billion). ${ }^{61}$ The number of people living with chronic pain in Australia is estimated to increase from 3.2 million in 2007 to 5.0 million in $2050 .^{62}$ Much of this persistent pain would be spinal pain.

A major reason for escalating health care expenditure relates to treatment and investigation cost inflation - annual expenditure for spinal pain management in 1995 in the United States was calculated to be US\$7.3 billion, whereas in 2007 the cost for drugs had skyrocketed some $271 \%$ to US $\$ 19.8$ billion, accounting for a sizable $23 \%$ of total direct health care expenditures. These trends are being reflected in Australia. ${ }^{63}$ Major elements accounting for this increase include the wider use of expensive drugs, spinal injections, ${ }^{64,65}$ and/or unnecessary investigations.

A systematic review of the cost-effectiveness of guideline-endorsed treatments for low back pain, in which 26 studies were appraised, ${ }^{66}$ demonstrated that spinal manipulation, interdisciplinary rehabilitation, exercise, acupuncture, or cognitive behavioral therapy all were cost-effective in individuals with subacute or chronic low back pain, while no evidence was found in support of medications, yoga, or relaxation. ${ }^{66}$ Furthermore, the same study indicated that care from a general practitioner did not appear to be the most costeffective means for managing low back pain, considering that adding spinal manipulation, exercise, behavioral counseling, and education/advice were more cost-effective than usual care from a general practitioner alone. ${ }^{66}$ Another systematic review by Michaleff et a ${ }^{67}$ supports the cost-effectiveness of spinal manipulative therapy, either alone or in combination with other treatment approaches. A convincing example of how a multidisciplinary, multimodal approach is likely to be more cost-effective than "standard" care for persistent pain is highlighted by the results obtained by Lin et al ${ }^{64}$ showing that a package of evidence-based treatments, which included spinal manipulation, is cost-effective for subacute and chronic low back pain and at least as cost-effective as other forms of conservative treatment. Recent Workers' Compensation data from USA suggest that patients with occupational spinal injuries visiting a surgeon first are significantly more likely to receive spinal surgery $(42.7 \%)$ than those whose first visit was with a musculoskeletal clinician $(1.5 \%){ }^{68}$ This association holds true even when controlling for injury severity and other measures, implying a significant cost saving and emphasizing the importance of fast access to appropriate assessment and care, so that best practice care may commence at an early stage of the disorder. Importantly, on synthesis of the outcomes of these studies, we do not suggest that any specific treatment should necessarily be viewed as inferior, but rather the importance of accurate triage and diagnosis, followed by appropriate care, is emphasized.

\section{Care approach and avoiding chronicity}

Research evidence supports the early referral and assessment of spinal pain patients by an appropriate health care practitioner, this may be a general practitioner or pain physician, but in the future may also include other trained and vetted clinicians, such as clinical nurse specialists, chiropractors, osteopaths, physician assistants, and musculoskeletal physiotherapists, with a view to offer treatment, facilitate health promotion, support rehabilitation, and offer patient education, ie, to apply the right treatment at the right time and in the right place. ${ }^{69}$ Early referral and assessment also has potential cost savings by avoiding unnecessary imaging/ investigations, hospitalizations, medical procedures, and surgery. ${ }^{70}$ Needless to say, health care policy- and decisionmakers would be very interested in any approach that may save on costs, which could be as much as a $20 \%$ saving on current expenditure for low back pain care within the mainstream health care sector. ${ }^{63}$

The importance of early access to appropriate care cannot be underestimated, since the aim of appropriate care is to alter the course of the disorder, particularly since low back pain is well-known to be either episodic or progress to chronicity. ${ }^{71}$ 
Hestbaek et $\mathrm{al}^{72,73}$ indicated that low back pain has an episodic trend in up to $80 \%$ of cases, as opposed to resolving fully, and Henschke ${ }^{74}$ reported that up to $30 \%$ of acute back pain becomes chronic. Around $25 \%$ of Australians who experience low back pain continue to have persistent or recurrent episodic back pain. ${ }^{75}$ Indeed, in a cohort of patients with acute low back pain in Australian primary care, prognosis was not as favorable as claimed in clinical guidelines - recovery was slow for most patients and nearly $33 \%$ of patients did not recover from the presenting episode, implying chronicity and added health care costs. ${ }^{74}$

Appropriately trained health care professionals could facilitate access to care at a community level and also identify predictors of chronicity in affected patients, ${ }^{71}$ which could subsequently be addressed through health/lifestyle modification and utilization of local healthy lifestyle programs, and could be as simple as a 20-40-minute walk a few times per week. $^{76,77}$ For example, a package of care using the latest evidence-based management, including patient education, staying active, exercise including a daily walk, lifestyle modification, spinal manipulative therapy, and simple analgesia, is likely to yield the best possible outcomes. ${ }^{24}$ This "package of care" approach would be particularly useful if combined with existing and currently funded programs focusing on lifestyle change and chronic pain prevention, such as the (Australian) Medicare Local (now Primary Care Network) Healthy Lifestyle and Chronic Pain Program and the SelfTraining Educative Pain Sessions (STEPS) program, with the goal of preventing chronicity. ${ }^{38,49,78}$

Short-term or periodic use of simple analgesia for mildto-moderate acute spinal pain (paracetamol, nonsteroidal anti-inflammatory drugs [NSAIDs]), and weak opioids such as tramadol for acute, moderate-to-severe spinal pain of less than 2 weeks is clinically defendable, notwithstanding recent evidence concerning the effectiveness ${ }^{79}$ and toxicity $^{80}$ of paracetamol. Some clinical guidelines recommend the limited use of strong opioids such as buprenorphine, morphine, and oxycodone, as the evidence of effectiveness is low (Level C), ${ }^{37}$ while the potential for harm is real. The use of benzodiazepines which interfere with memory should be limited. The guidelines support the use of paracetamol, NSAIDs (less than 3 months), and weak opioids, such as tramadol, in combination with paracetamol. ${ }^{37}$

The Musculoskeletal Analgesic Regime to Aid Rehabilitation (MARTAR) regime, developed by the WA Emergency Medicine Research Online (WAEMRO), suggests a graded approach to prescribing opioid analgesics is recommended based on the severity of the back pain (usually presenting in the emergency department as severe, acute back pain) over the short-term (short-term implying 2 weeks or less). ${ }^{81}$ Depending on the severity of pain, the MARTAR regime recommends various analgesics, including oxycodone immediate-release (IR) and morphine, alongside benzodiazepines such as clonazepam and diazepam. The regime emphasizes, however, that evaluation of the person with pain should occur $\leq 4$ hourly and analgesics titrated accordingly. Outside of the acute hospital setting, we do not recommend the regular use of potentially addictive opioid analgesia, such as oxycodone, pethidine, or morphine, for severe pain, but rather less addictive analgesics, such as tramadol (Schedule 4, prescription only medication). If a strong opioid is considered, then buprenorphine (Schedule 8 , controlled medication, requiring prescription and the prescribing is audited) is the least harmful effective option ${ }^{82,83}$ as it is the only strong opioid not associated with rapid tolerance, opioid-induced hyperalgesia, ${ }^{84}$ lowering of the person's sex hormones, ${ }^{85}$ or a negative impact on the patient's immune system via inhibiting their natural killer cells. In addition, buprenorphine has both an analgesic and antihyperalgesic effect ${ }^{82}$ which is relevant in neuropathic pain, which can be a contributor in spinal pain. ${ }^{86}$

Strong opioid drugs (S8) and benzodiazepines are associated with much higher risks and complications related to tolerance, addiction, and abuse, particularly with chronic or recurrent spinal pain syndromes. ${ }^{87,88}$ The problems associated with opioid use seem to emerge predominantly outside of the acute hospital setting - prescriptions for oxycodone in Australia have increased by more than $150 \%$ in the 5-year period up to 2008, with 551 Australians dying as a result of accidental overdose of prescribed opioids in the same year. ${ }^{89}$ An estimated 1,300 Australians aged 15-54 years died from accidental overdose of prescribed opioids in 2009/2010 - "most of the existing guidelines have limited impact on what is now approaching a national epidemic". ${ }^{90}$ Except for the short-term treatment of acute, severe cases of back pain, where opioid and benzodiazepines are a defendable option, there is little evidence to suggest that full $\mu$-agonist opioids change the course or severity of back pain ${ }^{37}$ to date. Compounding the matter are patients who put their doctors under pressure to prescribe opioids, often leading general practitioners to overlook behavioral nonpharmacological clinical guideline recommendations for nonmalignant pain. ${ }^{91}$

By extrapolation, a key area for undergraduate training of health care professionals, especially pharmacists and doctors, would be to increase the knowledge with clinical guidelines, ${ }^{92}$ in the aim to increase guideline-concordant treatment. We advocate assessment and treatment of the person with spinal pain by an 
appropriate, vetted, trained guideline-concordant health care professional, with a view to triage, diagnose, and manage acute, mild-moderate spinal pain and attempt to prevent chronicity.

\section{Health care politics and available workforce}

The immense burden and cost of conditions resulting in persistent pain in Australia is eloquently summarized by Briggs et al..$^{93}$ There are various journal editorials that suggest a bumpy road ahead regarding health care funding, a common theme across the globe, and ongoing concerns about how politicians and health care decision makers will define or determine "health". ${ }^{94}$ Therefore, when making recommendations about care in any future model or framework of care, it will be imperative that resources (sustainable funding, access to care, and workforce capacity) become the fourth principle, or "leg", of evidence-based practice, receiving equal attention in clini$\mathrm{cal}$, academic, and policy decision-making. Any recommended evidence-based treatment simply cannot be delivered if the resources and sustainable funding is not forthcoming.

Health care workforce analysis by the Australian Productivity Commission highlighted the desirability of "task substitution" $" 95$ and a recent new-graduate health care practitioner survey identified emerging health care workforce capacity that could cater for the multidisciplinary community-based approach for nonmalignant spinal pain, ${ }^{92}$ with the proviso that the health care professionals were trained, and potentially monitored, for clinical guideline concordant care. In particular, appropriately trained health care professionals such as clinical nurse specialists, chiropractors, osteopaths, physician assistants, and musculoskeletal physiotherapists, in addition to the traditional practitioners engaging with pain management, could be counted as part of the health care workforce. These additional health care disciplines would be able to fill some of the workforce gaps.

\section{Goals and process of spinal pain management}

MoC (Model of Care) can help to address the burden of service gaps in musculoskeletal health. ${ }^{30} \mathrm{An} \mathrm{MoC}$ is an evidence-based strategy, framework, or pathway that outlines the optimal manner in which care for specific types or groups of conditions should be made available and delivered to consumers. An MoC aims to

... include contemporary evidence with a framework to meet the current and projected community needs, within the context of local operational requirements. ${ }^{93}$
Importantly, an $\mathrm{MoC}$ is not an clinical practice guideline. ${ }^{30}$ Attention needs to be given to not only effective evidence-based management but also to other factors, such as poor lifestyle, lack of exercise, and patient education. In addition, management of persistent and/or complex spinal pain requires timely follow-up and ongoing consumer participation in their care, which usually needs ongoing supervision, ie, coaching. Management of a person with pain is, therefore, a series of ongoing activities that requires continuing input and participation by both the consumer and the multidisciplinary health care team, respectively. Guidelines on these activities would be included in an $\mathrm{MoC}$ document, thereby offering an outline of consumer's care journey.

The goals, then, of an evidence-based $\mathrm{MoC}$ would be to:

1. Broaden care services to span from hospital-based to community-based services with a view to improve and facilitate earlier access to care, embrace a wider relevant workforce, and contain costs by attempting to avoid hospitalization, unnecessary investigations, and possibly long waiting times for appointments;

2. Expand care services across hospital-based and community-based services with a view to improve and facilitate earlier access to care, embrace a wider relevant workforce, and contain costs by attempting to avoid hospitalization, unnecessary investigations, and long waiting times for specialist appointments;

3. Orchestrate a multidisciplinary approach to care, thereby offering care tailored to the individual needs of the person with pain, which is likely to produce the best possible clinical outcomes. Depending on the complexity of the case, this may include referral to a secondary or tertiary hospital for procedures and/or relevant investigations;

4. Facilitate quicker and early access of persons with spinal pain to assessment and appropriate care, guided by triage and diagnosis, so to reduce the duration of morbidity and attempt to avoid chronicity of pain. Avoiding, or at least managing, persistent pain would likely have significant cost savings; and

5. Disseminate information and educate a) the public, b) consumers, and c) health care professionals regarding the best practice management of spinal pain.

Care services for the management of persons with pain, as presented in an MoC or Care Framework document, would include:

1. Flow charts and diagrams that provide an indication of the nature and levels of care expected, starting with a triage process of a person with pain by a vetted, credentialed 
health care practitioner, followed by treatment and/or referral to another care provider or service, and so on;

2. A clear, pragmatic representation of the levels of care matched with the case complexity, with an explanation of each level of care;

3. An outline of the consumer's care journey with information on accessibility to care services, relevant organizations, and self-help material;

4. Rationalize the description care services and the complex process of care service delivery in an MoC document so that end users may easily grasp 1) the concept of spinal pain, and 2) how, who, and where to access the care services.

For example, uncomplicated cases of persons with spinal pain may be triaged and managed by the general practitioner or other vetted health care provider in the community, without the need for referral elsewhere. For more complex cases, or where preventing the persistence of pain is a priority, the primary contact health care provider would triage the "whole" patient and refer to a care service or appropriate provider - this triage service would include assessment and determination of the likely level of care needed based on the complexity of the case. At triage, the consumer would also receive appropriate information and education about pain, as this appears to be important in positively changing consumers' attitudes, expectations, and beliefs..$^{96,97}$

\section{Workforce capacity and care teams}

To offer coordinated multimodal care, health care teams are recommended, consisting of various disciplines depending on the level of care an individual person with spinal pain requires. To determine the level of care needed, the person with pain would go through a triage process, and, where necessary, result in a referral to the appropriate health care provider or multidisciplinary team for further care. This may include the referral to a secondary or tertiary hospital.

The purpose of creating care teams would be to include health care disciplines that:

1. Would make a meaningful contribution to the care of a person with spinal pain, be it via case management, treatment, or assessment;

2. Facilitate task substitution where various disciplines may be involved in patient triage, case management, treatment, and coordination of care;

3. Offer cost-efficient, evidence-based interventions that, as a package of care, would offer the best possible outcomes and avoid unnecessary interventions, investigations, hospital admissions, or duplication of care;
4. Promote early assessment, triage, care, and referral of persons with pain, with a view to avoid chronicity of symptoms by offering the right treatment by the right discipline(s) at the right time.

Care teams would be created through a process of identifying vetted disciplines that are associated with spinal pain and/or pain management. In some cases, task substitution would be feasible where triage and assessment of patients may be completed by those other than the traditional health care gatekeeper(s). Appropriate health care practitioners would be certified to work as part of a multidisciplinary team with a view to offer coordinated care based on the recommendations of a care framework or MoC. These health care teams, as part of their involvement in audit and evaluation of their services, should engage with academics/researchers with an interest in pain health, with a view to develop research projects that examine, explore, and test care services for spinal pain.

Teamwork cannot be emphasized enough. We acknowledge that, in a competitive health care marketplace, practitioners become protective over their "patch" and become adversarial when there is a potential threat to their income and/or professional authority - this is natural. We take this opportunity to point out that collaboration and teamwork is likely to generate more business via referrals and ease of access for consumers. We draw upon observations and experience from the business management sector, where developing trusting and collaborative networks or teams yields better results than adversarial relationships or overt competition. ${ }^{98}$

\section{Future direction of research}

No doubt, there is an ongoing need to explore the complex nature of pain and pain states, which includes spinal pain, with a view to gain further insight into appropriate management. Research efforts should align with health care needs and, with the principles of evidence-based practice in mind, should endeavor to inform clinical guidelines, $\mathrm{MoC}$ and policy. ${ }^{47}$ Future definitive studies, for example prospective cohort studies or cluster analyses, are more likely to be valuable in establishing the effectiveness of multimodal, complex interventions in specific clinical environments.

We feel that research should, in the context of models or frameworks of care, be directed toward:

1. The testing of complex interventions, in the form of care packages, protocols, or combinations of treatment, using well-designed and piloted clinical trials. This form of research would test care services, as opposed to 
individual treatments. Outcomes of such research projects would inform practice and keep clinical guidelines/MoC up to date;

2. Conducting practice-based cohort studies that gather data on patient and practice characteristics, so as to gain insight into the demographics of persons with pain, workforce capacity, and, possibly, clinical outcomes. Collected data would inform the need for workforce expansion, emerging resource requirements, and assist in updating care service policy alongside providing a clear description of consumer preference(s);

3. Doing translational and integrative research that facilitates the development and testing of mechanisms that try to get research into practice and policy. Also, such research will provide insight on how to facilitate the adherence of health care stakeholders to policy, clinical guidelines, and MoC. The positive consequence of such research is likely to be major cost savings and promotion of best practice;

4. Address the gap in current high-level research, which tends to exclude the complex patient, especially if they have associated mental health issues, including anxiety, stress, and depression, or if they are at the extremes of age such as children, adolescents, and elder patients (greater than 65 years of age);

5. Allow exploration of new or refined pain management techniques, with an active audit process that financially supports the validation processes; and

6. Prioritize funding for clinical research that has ongoing cohorts of real patients presenting at the clinical interface for treatment for significant pain.

In our view, research organizations should continue to develop research agendas that align with the direction of research stated above, while simultaneously considering available research funding opportunities that match those health care priorities. An example of very informative and influential research is the long-standing, continuous cross-sectional Australian BEACH (Bettering the Evaluation And Care of Health) program, ${ }^{99,100}$ which collects data on encounters, practitioners, and patients in general practice. These data have had a tremendous impact on informing practice and policy. Another example is the ACORN (Australian Chiropractic Research Network) project, ${ }^{101}$ a longitudinal practice-based study, which has attracted over 1,600 practitioners, with a view to collect practice and patient data. Such data, alongside those already collected, ${ }^{102}$ will serve to describe the various health care disciplines linked with spinal health and clarify the roles of these disciplines. Research efforts and data should be amalgamated or grouped where possible, in the form of meta-analytical reviews. Again, the proviso in reviewing grouped data is the negative published bias that can overcall the effectiveness of an intervention, as the tendency is to publish positive outcome trials. The clinical trials registers are enabling protocol registration world-wide and contact between research groups. ${ }^{103}$ Grouping research efforts and data meta-analysis would make outcomes or clinical trials more generalizable.

A paradox is that current funding for pain research by, for example, the National Institute of Health (USA) is $<1 \%$ of the overall ${ }^{104}$ spend on research. This is minimal when compared to the actual cost of care for pain management. Strong research leadership is warranted, particularly during times of cost constraints and tight budgets, because clinical research can often be viewed as a nonessential expense by health care administrators and even clinicians, ${ }^{105}$ where service delivery is considered to be of higher priority. Not only is ongoing research critical for obtaining data on outcomes and patient/practice characteristics, but it also serves to inform and improve on guidelines and practice. Therefore, both ongoing research and the training of talented clinical researchers will be important in ensuring improvement in patient care. Creating and maintaining additional clinical professor positions, would facilitate the tripartite goal of research, teaching, and integration of research into clinical practice.

\section{Conclusion}

We suggest that there is sufficient understanding of nonmalignant spinal pain to be able to develop and implement care frameworks or $\mathrm{MoC}$ that are based on the principles of evidence-based practice, matched with available funding and resources. A multidisciplinary approach tailored to the individual patient care needs, delivered at an appropriate level of care by health care teams, is recommended to achieve the best possible outcomes, improve on patient satisfaction with care, and be cost efficient. Leadership, ongoing research and continuing advancement of $\mathrm{MoC}$ is needed to improve the care services for spinal pain.

\section{Disclosure}

The authors report no conflicts of interest in this work.

\section{References}

1. Hurwitz EL. Commentary: exercise and spinal manipulative therapy for chronic low back pain: time to call for a moratorium on future randomized trials? Spine J. 2011;11(7):599-600. 
2. New Zealand Guidelines Group. Available from: http://www.nzgg.org. nz/guidelines/acutenslbp. Accessed June 6, 2015.

3. Clinical Practice Guidelines Portal. Available from: https://www.clinicalguidelines.gov.au/. Accessed June 6, 2015.

4. Low Back Pain: Guidelines for its Management. Available from: http:// www.backpaineurope.org/. Accessed June 6, 2015.

5. Rubinstein SM, van Middelkoop M, Assendelft WJ, de Boer M, van Tulder M. Spinal manipulative therapy for chronic low-back pain. Cochrane Database Syst Rev. 2011;2:CD008112.

6. van Middelkoop M, Rubinstein SM, Verhagen AP, Ostelo RW, Koes BW, van Tulder MW. Exercise therapy for chronic nonspecific low-back pain. Best Pract Res Clin Rheumatol. 2010;24(2):193-204.

7. Koes B, van Tulder M, Lin C, Macedo L, McAuley J, Maher C. An updated overview of clinical guidelines for the management of non-specific low back pain in primary care. Eur Spine J. 2010;19: 2075-2094.

8. Henschke N, Ostelo RW, van Tulder MW, et al. Behavioural treatment for chronic low-back pain. Cochrane Database Syst Rev. 2010;7:CD002014.

9. Moseley GL. Widespread brain activity during an abdominal task markedly reduced after pain physiology education: fMRI evaluation of a single patient with chronic low back pain. Aust J Physiother. 2005;51(1):49-52.

10. McGuirk B, Bogduk N. Evidence-based care for low back pain in workers eligible for compensation. Occup Med. 2007;57(1):36-42.

11. Mercado AC, Carroll LJ, Cassidy D, Cote P. Passive coping is a risk factor for disabling neck or low back pain. Pain. 2005;117(1-2):51-57.

12. Karjalainen K, Malmivaara A, van Tulder MW, et al. Multidisciplinary biopsychosocial rehabilitation for subacute low-back pain among working age adults [last substantial update]. Cochrane Database Syst Rev. 2003;(2):CD002193.

13. Damush TM, Weinberger M, Perkins SM, et al. The long-term effects of a self-management program for inner-city primary care patients with acute low back pain. Arch Intern Med. 2003;163(21):2632-2638.

14. Turner JA, Clancy S. Comparison of operant behavioral and cognitivebehavioral group treatment for chronic low back pain. $J$ Consult Clin Psychol. 1988;56(2):261-266.

15. Koes B, van Tulder M, Ostelo R, Kim Burton A, Waddell G. Clinical guidelines for the management of low back pain in primary care: an international comparison. Spine. 2001;26(22):2504-2513.

16. van Tulder MW, Koes BW, Bouter LM. Conservative treatment of acute and chronic nonspecific low back pain: a systematic review of randomized controlled trials of the most common interventions. Spine. 1997;22(18):2128-2156.

17. Borghouts JA, Koes BW, Bouter LM. The clinical course and prognostic factors of non-specific neck pain: a systematic review. Pain. 1998;77(1):1-13.

18. Bronfort G, Haas M, Evans RL. Efficacy of spinal manipulation and mobilization for low back pain and neck pain: a systematic review and best evidence synthesis. Spine J. 2004;4(3):335-356.

19. Gross AR, Kay T, Hondras M, et al. Manual therapy for mechanical neck disorders: a systematic review. Man Ther. 2002;7(3):131-149.

20. Leboeuf-Yde C, Fejer R, Nielsen J, Kyvik K, Hartvigsen J. Pain in the three spinal regions: the same disorder? Data from a populationbased sample of 34,902 Danish adults. Chiropr Man Therap. 2012; 20(1):11.

21. Harvey E, Burton A, Klaber-Moffett J, Breen A. Spinal manipulation for low-back pain: a treatment package agreed by the UK chiropractic, osteopathy and physiotherapy professional associations. Man Ther. 2003;8:46-51.

22. Ruoff GE, Rosenthal N, Jordan D, Karim R, Kamin M. Tramadol/ acetaminophen combination tablets for the treatment of chronic lower back pain: a multicenter, randomized, double-blind, placebo-controlled outpatient study. Clin Ther. 2003;25(4):1123-1141.

23. Guzman J, Esmail R, Karjalainen K, MalmivaaraA, Irvin E, Bombardier C. Multidisciplinary rehabilitation for chronic low back pain: systematic review. BMJ. 2001;322(7301):1511-1516.
24. Parkin-Smith GF, Norman IJ, Briggs E, Angier E, Wood TG, Brantingham JW. A structured protocol of evidence-based conservative care compared with usual care for acute nonspecific low back pain: a randomized clinical trial. Arch Phys Med Rehabil. 2012;93(1):11-20.

25. Carey TS, Garrett J, Jackman A, McLaughlin C, Fryer J, Smucker DR. The outcomes and costs of care for acute low back pain among patients seen by primary care practitioners, chiropractors, and orthopedic surgeons. The North Carolina Back Pain Project. N Engl J Med. 1995; 333(14):913-917.

26. Froud R, Eldridge S, Lall R, Underwood M. Estimating the number needed to treat from continuous outcomes in randomised controlled trials: methodological challenges and worked example using data from the UK Back Pain Exercise and Manipulation (BEAM) trial. BMC Med Res Methodol. 2009;9(1):35.

27. Nielson WR, Weir R. Biopsychosocial approaches to the treatment of chronic pain. Clin J Pain. 2001;17(4):S114-S127.

28. Hancock MJ, Maher CG, Laslett M, Hay E, Koes B. Discussion paper: what happened to the 'bio' in the bio-psycho-social model of low back pain? Eur Spine J. 2011;20(12):2105-2110.

29. Speerin R, Slater H, Li L, et al. Moving from evidence to practice: models of care for the prevention and management of musculoskeletal conditions. Best Pract Res Clin Rheumatol. 2014;28(3): 479-515.

30. Medical Workforce Report 2013/14, Profiling, Performance, Programs and Priorities: Office of the Chief Medical Officer, Department of Health, Western Australia, 2015. Available from: http://ww2.health. wa.gov.au/ /media/Files/Corporate/Reports\%20and\%20publications/ Medical\%20Workforce/Medical-Workforce-Report-201314.ashx. Accessed June 6, 2015.

31. National Health and Medical Research Council. Annual Report on Australian Clinical Practice Guidelines. Canberra, Australia: National Health and Medical Research Council; 2014. Available from: http:// www.nhmrc.gov.au/guidelines-publications/nh165. Accessed August 4, 2015.

32. Amorin-Woods LG, Beck RW, Parkin-Smith GF, Lougheed J, Bremner AP. Adherence to clinical practice guidelines among three primary contact professions: a best evidence synthesis of the literature for the management of acute and subacute low back pain. J Can Chiropr Assoc. 2014;58(3):220-237.

33. Reddy S, Herring SE. Can Australia's clinical practice guidelines be trusted? Med J Aust. 2015;202:8.

34. Pronovost PJ. Enhancing physicians' use of clinical guidelines. JAMA. 2013;310(23):2501-2502.

35. Underwood MR, Morton V, Farrin A. Do baseline characteristics predict response to treatment for low back pain? Secondary analysis of the UK BEAM dataset [ISRCTN32683578]. Rheumatology. 2007;46(8): 1297-1302.

36. Bishop P, Quon J, Olson D, et al. The CHIRO study (Chiropractic Hospital-based Interventions Research Outcomes) clinical practice guidelines for medical and chiropractic care of acute lower back pain: a randomized controlled trial. Spine. 2007;7(5 Suppl 1):11S.

37. Airaksinen O, Brox J, Cedraschi C, et al. COST B13 European guidelines for the management of chronic non-specific low back pain. Eur Spine J. 2006;15:S192-S300.

38. Goertz CM, Long CR, Hondras MA, et al. Adding chiropractic manipulative therapy to standard medical care for patients with acute low back pain: results of a pragmatic randomized comparative effectiveness study. Spine. 2013;38(8):627-634.

39. Hay E, Mullis R, Lewis M, et al. Comparison of physical treatments versus a brief pain-management programme for back pain in primary care: a randomised clinical trial in physiotherapy practice. Lancet. 2005;365:2024-2030.

40. Christelis N, Wallace S, Sage CE, et al. An enhanced recovery after surgery program for hip and knee arthroplasty. Med JAust. 2015;202(7): 363-368.

41. Flor H, Fydrich T, Turk D. Efficacy of multidisciplinary pain treatment centers: a meta-analytic review. Pain. 1992;49(2):221-230. 
42. Patrick LE, Altmaier EM, Found EM. Long-term outcomes in multidisciplinary treatment of chronic low back pain: results of a 13-year follow-up. Spine. 2004;29(8):850-855.

43. Monticone M, Ambrosini E, Rocca B, Magni S, Brivio F, Ferrante S. A multidisciplinary rehabilitation programme improves disability, kinesiophobia and walking ability in subjects with chronic low back pain: results of a randomised controlled pilot study. Eur Spine J. 2014;10:2105-2113.

44. Moradi B, Hagmann S, Zahlten-Hinguranage A, et al. Efficacy of multidisciplinary treatment for patients with chronic low back pain: a prospective clinical study in 395 patients. J Clin Rheumatol. 2012;18(2):76-82.

45. Jensen CE, Riis A, Pedersen KM, Jensen MB, Petersen KD. Study protocol of an economic evaluation of an extended implementation strategy for the treatment of low back pain in general practice: a cluster randomized controlled trial. Implement Sci. 2014;9:140.

46. Petit A, Roche-Leboucher G, Bontoux L, et al. Effectiveness of three treatment strategies on occupational limitations and quality of life for patients with non-specific chronic low back pain: is a multidisciplinary approach the key feature to success: study protocol for a randomized controlled trial. BMC Musculoskelet Disord. 2014;15:131.

47. Sackett DL, Rosenberg WMC, Muir Gray JA, Haynes RB, Richardson WS. Evidence based medicine: what it is and what it isn't. BMJ. 1996; 312(7023):71-72.

48. Bonica J. The need of a taxonomy. Pain. 1979;6(3):247-248.

49. Davies S, Quintner J, Parsons R, et al. Preclinic group education sessions reduce waiting times and costs at public pain medicine units. Pain Med. 2011;12(1):59-71.

50. Davies S. Whole person engagement for the treatment of people in pain. Medicus. 2015;55(2):23.

51. Karjalainen K, Malmivaara A, van Tulder M, et al. Multidisciplinary biopsychosocial rehabilitation for subacute low back pain in working-age adults: a systematic review within the framework of the Cochrane Collaboration Back Review Group. Spine. 2001;26(3): 262-269.

52. Foster N, Delitto A. Embedding psychosocial perspectives within clinical management of low back pain: integration of psychosocially informed management principles into physical therapist practicechallenges and opportunities. Phys Ther. 2011;91(5):790-803.

53. Pincus T, Burton AK, Vogel S, Field AP. A systematic review of psychological factors as predictors of chronicity/disability in prospective cohorts of low back pain. Spine. 2002;27(5): E109-E120.

54. Grotle M, Foster NE, Dunn KM, Croft P. Are prognostic indicators for poor outcome different for acute and chronic low back pain consulters in primary care? Pain. 2010;151(3):790-797.

55. Froud R, Patterson S, Eldridge $\mathrm{S}$, et al. A systematic review and meta-synthesis of the impact of low back pain on people's lives. $B M C$ Musculoskelet Disord. 2014;15(1):50.

56. Davies S. Pain and Modern Medicine: Presentation Consumer Forum: STEPS in Darwin, 2011. Available from: http:/www.slideshare.net/ ArthritisNT/pain-and-modern-medicine-stephanie-davies. Accessed June 8, 2015.

57. Slater H, Briggs AM, Bunzli S, Davies SJ, Smith AJ, Quintner JL. Engaging consumers living in remote areas of Western Australia in the self-management of back pain: a prospective cohort study. BMC Musculoskelet Disord. 2012;13(69):1471-2474.

58. Salomon JA, Vos T, Hogan DR, et al. Common values in assessing health outcomes from disease and injury: disability weights measurement study for the Global Burden of Disease Study 2010. Lancet. 2012;380(9859):2129-2143.

59. Horton R. GBD 2010: understanding disease, injury, and risk. Lancet. 2012;380(9859):2053-2054

60. Hogg MN, Gibson S, Helou A, DeGabriele J, Farrell MJ. Waiting in pain: a systematic investigation into the provision of persistent pain services in Australia. Med J Aust. 2012;196(6):386-390.
61. Access Economics. The high price of pain: the economic impact of persistent pain in Australia in 2007. Report by Access Economics Pty Limited for MBF Foundation in collaboration with the University of Sydney, 2007. Available from: http:/www.bupa.com.au/staticfiles/ BupaP3/Health\%20and\%20Wellness/MediaFiles/PDFs/MBF_Founda tion_the_price_of_pain.pdf. Accessed 13 November, 2011.

62. Burke AL, Denson LA, Mathias JL, Hogg MN. An analysis of multidisciplinary staffing levels and clinical activity in Australian tertiary persistent pain services. Pain Med. 2015;16(6):1221-1237.

63. Martin BI, Deyo RA, Mirza SK, et al. Expenditures and health status among adults with back and neck problems. JAMA. 2008;299(6):656-664.

64. Zerzan J, Morden NE, Soumerai S, Ross-Degnan D, Roughead EE, Zhang F. Trends and geographic variation of opiate medication use in state Medicaid fee-for service programs,1996-2002. Med Care. 2006;44(11):1005-1010.

65. Friedly J, Chan L, Deyo R. Increases in lumbosacral injection in the Medicare population, 1994 to 2001. Spine. 2007;32(16):1754-1760.

66. Lin C, Haas M, Maher C, Machado L, van Tulder M. Cost-effectiveness of guidelines-endorsed treatments for low back pain: a systematic review. Eur Spine J. 2011;20(7):1024-1038.

67. Michaleff Z, Lin C, Maher C, van Tulder M. Spinal manipulation epidemiology: systematic review of cost effectiveness studies. J Electromyogr Kinesiol. 2012;22(5):655-662.

68. Keeney B, Fulton-Kehoe D, Turner J, et al. Early predictors of lumbar spine surgery after occupational back injury: results from a prospective study of workers in Washington state. Spine. 2013;38(11):953-964.

69. NHS (UK). Right Care, Right Place, 2011. Available from: http://www. rightcarerightplace.nhs.uk/page.asp?fldArea=0\&fldMenu=0\&fldSubM enu $=0 \&$ fldKey=1. Accessed June 6, 2015.

70. Leape LL. Unnecessary surgery. Annu Rev Public Health. 1992;13: 363-383.

71. Klenerman L, Slade PD, Stanley IM, et al. The prediction of chronicity in patients with an acute attack of low back pain in a general practice setting. Spine. 1995;20(4):478-484.

72. Hestbaek L, Leboeuf-Yde C, Engberg M, Lauritzen T, Bruun NH, Manniche $\mathrm{C}$. The course of low back pain in a general population. Results from a 5-year prospective study. J Manipulative Physiol Ther. 2003;26(4):213-219.

73. Hestbaek L, Leboeuf-Yde C, Manniche C. Low back pain: what is the long-term course? A review of studies of general patient populations. Eur Spine J. 2003;12:149-165.

74. Henschke N. Prognosis in patients with recent onset of low back pain in Australian primary care: inception cohort study. BMJ. 2008;337:a171.

75. Walker BF, Muller R, Grant WD. Low back pain in Australian adults. Health provider utilization and care seeking. J Manipulative Physiol Ther. 2004;27(5):327-335.

76. Sitthipornvorakul E, Janwantanakul P, Lohsoonthorn V. The effect of daily walking steps on preventing neck and low back pain in sedentary workers: a 1-year prospective cohort study. Eur Spine J. 2015;24(3):417-424.

77. Shnayderman I, Katz-Leurer M. An aerobic walking programme versus muscle strengthening programme for chronic low back pain: a randomized controlled trial. Clin Rehabil. 2013;27(3):207-214.

78. Self Training Educative Pain Sessions (STEPS) Program. Perth North Metro Medicare Local. Available from: http://www.pnml.com.au/ medicare-local-programs/68-programs/article/178-steps. Accessed May 11, 2015.

79. Williams CM, Maher CG, Latimer J, et al. Efficacy of paracetamol for acute low-back pain: a double-blind, randomised controlled trial. Lancet. 2014;384(9954):1586-1596.

80. Machado GC, Maher CG, Ferreira PH, et al. Efficacy and safety of paracetamol for spinal pain and osteoarthritis: systematic review and meta-analysis of randomised placebo controlled trials. BMJ. 2015;350:h1225.

81. ClinicalNotes.net (Western Australian Emergency Medicine Research Online). Available from: https://clinicalnotes.net/martar-faq. Accessed May 11, 2015. 
82. Hans G. Buprenorphine - a review of its role in neuropathic pain. J Opioid Manag. 2007;3(4):195-206.

83. Gordon A, Callaghan D, Spink D, et al. Buprenorphine transdermal system in adults with chronic low back pain: a randomized, doubleblind, placebo-controlled crossover study, followed by an open-label extension phase. J Clin Ther. 2010;32(5):844-860.

84. Koppert W, Ihmsen H, Körber N, et al. Different profiles of buprenorphine-induced analgesia and antihyperalgesia in a human pain model. Pain. 2005;118(1-2):15-22.

85. Bliesener N, Albrecht S, Schwager A, Weckbecker K, Lichtermann D, Klingmuller D. Plasma testosterone and sexual function in men receiving buprenorphine maintenance for opioid dependence. J Clin Endocrinol Metab. 2005;90(1):203-206.

86. Freynhagen R, Baron R, Gockel U, Tölle TR. painDETECT: a new screening questionnaire to identify neuropathic components in patients with back pain. Curr Med Res Opin. 2006;22(10): 1911-1920.

87. Kahan M, Mailis-Gagnon A, Wilson L, Srivastava A. Canadian guideline for safe and effective use of opioids for chronic noncancer pain: clinical summary for family physicians. Part 1: general population. Can Fam Physician. 2011;57(11):1257-1266.

88. The Royal Australasian College of Physicians. Prescription Opioid Policy: improving management of chronic non-malignant pain and prevention of problems associated with prescription opioid use, Sydney, 2008. Available from: http://www.fpm.anzca.edu.au/resources/ professional-documents/documents/Prescription\%20Opioid\%20Policy. pdf. Accessed June 8, 2015.

89. Roxburgh A, Burns L. Accidental drug-induced deaths due to opioids in Australia, 2008. Sydney, NSW: National Drug and Alcohol Research Centre; 2012. Available from: http://ndarc.med.unsw.edu.au/resource/ accidental-opioid-induced-deaths-australia-2008.

90. Bramwel N. Call for incentive to tackle opioid 'epidemic'. Medical Observer. October 30, 2012. Available from: http://www. medicalobserver.com.au/news/call-for-incentive-to-tackle-opioidepidemic?utm_medium=email\&utm_campaign=Daily+Enews++31102012\&utm_content=Daily+Enews+-+31102012+CID_40e50d1 e792940dd1e3cf00a279e7ad0\&utm_source=Email\%20marketing\%20 software\&utm_term $=$ Call $\% 20$ for $\% 20$ incentive $\% 20$ to $\% 20$ tackle $\% 20$ opioid\%20epidemic. Accessed August 4, 2015.

91. Holliday S, Magin P, Dunbabin J, et al. An evaluation of the prescription of opioids for chronic nonmalignant pain by Australian general practitioners. Pain Med. 2013;14(1):62-74.

92. Briggs AM, Slater H, Smith AJ, Parkin-Smith GF, Watkins K, Chua J. Low back pain-related beliefs and likely practice behaviours among final-year cross-discipline health students. Eur J Pain. 2012;17(5): 766-775.
93. Briggs AM, Towler SC, Speerin R, March LM. Models of care for musculoskeletal health in Australia: now more than ever to drive evidence into health policy and practice. Aust Health Rev. 2014;38(4): 401-405.

94. Geelhoed E. Is our current health system sustainable? Medicus. 2015;55(1):35.

95. Productivity Commission. Australia's Health Workforce, Research Report. Canberra, Australia: Productivity Commission; 2005.

96. Darlow B, Dowell A, Baxter GD, Mathieson F, Perry M, Dean S. The enduring impact of what clinicians say to people with low back pain. Ann Fam Med. 2013;11(6):527-534.

97. Clarke KA, Iphofen R. Accepting pain management or seeking pain cure: an exploration of patients' attitudes to chronic pain. Pain Manag Nurs. 2007;8(2):102-110.

98. Masterson M. Ready, Fire, Aim: Zero to \$100 Million in No Time Flat. 1st ed. Hoboken, NJ: Wiley; 2008.

99. Britt H, Miller G, Henderson J, et al. General Practice Activity in Australia 2011-2012. General Practice Series Number 31. Sydney, NSW: Sydney University Press; 2012.

100. Harris MF, Furler J, Valenti L, Harris E, Britt H. Matching care to need in general practice: a secondary analysis of Bettering the Evaluation and Care of Health (BEACH) data. Aust J Prim Health. 2004;10(3):151-155.

101. Adams J. The ACORN Project, 2014. Available from: https://www. uts.edu.au/research-and-teaching/our-research/arccim/research/acornproject. Accessed December 30, 2014.

102. French S, Charity M, Forsdike K, et al. Chiropractic Observation and Analysis STudy (COAST): providing an understanding of current chiropractic practice. Med J Aust. 2013;199(10):687-691.

103. Ellis N, Johnston V, Gargett S, et al. Does self-management for return to work increase the effectiveness of vocational rehabilitation for chronic compensated musculoskeletal disorders? Protocol for a randomised controlled trial. BMC Musculoskelet Disord. 2010;11(115): 1471-2474.

104. Relieving Pain in America: A Blueprint for Transforming Prevention, Care, Education, and Research, 2011. Available from: https://www. iom.edu/Reports/2011/Relieving-Pain-in-America-A-Blueprint-forTransforming-Prevention-Care-Education-Research.aspx. Accessed 12 May, 2015.

105. Allison S, Bastiampillai T, Baune BT. Enabling the success of academic health science centres in Australia: where is the leadership? Med $J$ Aust. 2015;202(9):475.
Journal of Pain Research

\section{Publish your work in this journal}

The Journal of Pain Research is an international, peer-reviewed, open access, online journal that welcomes laboratory and clinical findings in the fields of pain research and the prevention and management of pain. Original research, reviews, symposium reports, hypothesis formation and commentaries are all considered for publication.
Dovepress

The manuscript management system is completely online and includes a very quick and fair peer-review system, which is all easy to use. Visit http://www.dovepress.com/testimonials.php to read real quotes from published authors. 